Barrett oesophagus (BE) is the only known precursor of oesophageal adenocarcinoma (EAC), which is rapidly increasing in incidence. $\mathrm{BE}$ involves intestinal metaplasia - the replacement of squamous epithelium by columnar epithelium - in the distal oesophagus as an adaptive response to gastro-oesophageal reflux.

\title{
DIAGNOSIS
}

High-definition endoscopy is the gold standard for diagnosis, which is confirmed by histological evidence of intestinal metaplasia in oesophageal biopsy samples, although the precise criteria for both methods are debated. Endoscopy is indicated in individuals $>50$ years of age with new-onset symptoms of reflux, or at any age if these symptoms persist despite medical therapy or if alarm symptoms (such as weight loss, persistent vomiting and bleeding in the upper gastrointestinal tract) are present. Lifestyle modifications (such as dietary changes, exercise and reducing smoking) can reduce the incidence of $\mathrm{BE}$, as can pharmacological agents such as proton pump inhibitors, NSAIDs and statins, although long-term chemoprevention is not currently recommended owing to potential adverse effects. Screening by upper endoscopy is only recommended in patients with chronic, frequent gastrooesophageal reflux symptoms and several other risk factors.

\section{INTESTINAL METAPLASIA} BE epithelium has a glandular structure comprising crypts with mucin-secreting goblet cells. BE may form by transdifferentiation of squamous epithelial cells and/or transcommitment of various stem

$$
\text { cell populations. }
$$
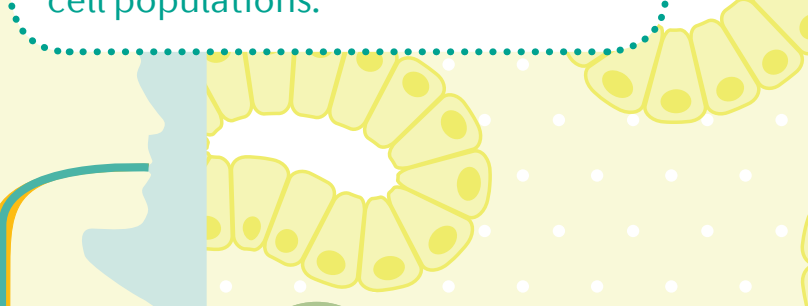

\section{EPIDEMIOLOGY}

The prevalence of $\mathrm{BE}$ is $\sim 1-2 \%$ in adults in Western countries, and the rate of progression to EAC is $0.1-0.5 \%$ per patient-year. The risk of developing BE is 2-3fold higher in white individuats than in other ethnicities and

\section{OESOPHAGEAL INJURY}

Exposure to gastric refluxate increases oxidative stress,

activates inflammatory mediators and induces DNA damage in oesophageal epithelial cells, leading to intestinal metaplasia.
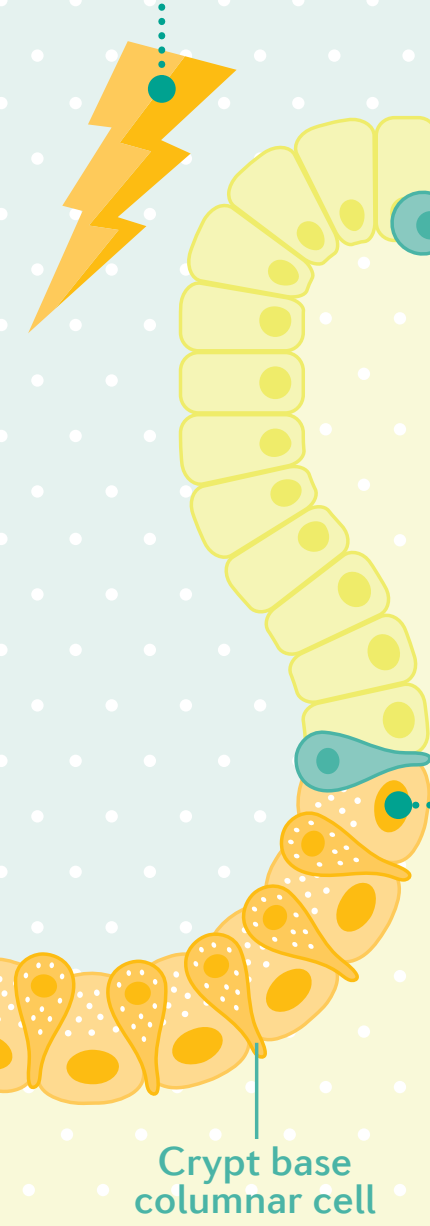

\section{ac00000}

90000

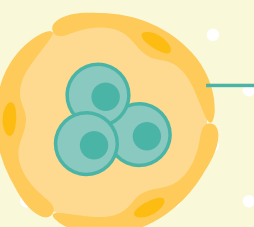

Blood vessel

\section{MALIGNANT PROGRESSION}

BE progression to dysplasia and

EAC involves TP53 mutations,

followed by oncogene

amplification and genomic

instability. Large genomic

alterations or genome duplication

can accelerate progression.

For the Primer visit doi:10.1038/s41572-019-0086-z

\section{MANAGEMENT}

Histological staging of disease is crucial to re, whereas endoscopic treatment is indicated 作 d . endoscopic mucosal resection is indicated, whereas the more technically demanding endoscopic submucosal dissection is only tranted for bulky lesions or suspected

After resection of neoplastic
lesions, the remaining BE
epithelium is removed
by endoscopic ablation.
Frequent recurrence of
BE and dysplasia after
endoscopic treatment
mandates regular
endoscopic surveillance.

OUTLOOK

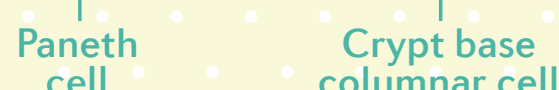

twofold hightrer in men than it: vomen. Age is also an important risk factor, with BE prevalence increasing by $50-100 \%$ with Every decade of life in adults. BE risk may also have a genetic component, although shared.

列

I Gastro-oesophageal reflux disease (GERD) age, sex, ethnicity, diet, obesity and smoking are important risk factors for develooment and progression of $\mathrm{BE}$

lifestyle elements and increased endoscopic screening in family members of individuals with gastro-oesophageal reftux disease (GERD) are onfounding factors.
The majority of patients with chronic GERD symptoms do not develop BE and most BE cases do not progress to EAC, so further research is needed to understand the molecular mediators of intestinal metaplasia and the inflammatory processes that contribute to $\mathrm{BE}$ development and malignant progression. Objective criteria for histological diagnosis of dysplasia are needed, as are better risk stratification tools for selecting populations for screening, which should aid in early diagnosis of $\mathrm{BE}$ and dysplasia, thereby improving outcomes. To improve surveillance in patients with $\mathrm{BE}$, additional risk factors for malignant progression need to be identified, which might also forego unnecessary surveillance and intervention in patients at low risk. 